\title{
Спектральные свойства флуктуаций концентрационного поля в электромембранных системах с сульфокатионообменными мембранами с разной дисперсностью ионообменника
}

\author{
Акберова Э.М. \\ ФГБОУ ВО «Воронежский государственный университет», Воронеж
}

Поступила в редакцию 10.11.2018 г.

DOI: https://doi.org/10.17308/sorpchrom.2018.18/624

\begin{abstract}
Методом Фурье-анализа определен спектральный состав флуктуаций концентрационного поля в стратифицированных системах с экспериментальными гетерогенными сульфокатионообменными мембранами Ralex CM Pes («MEGA» a.s., Чехия), содержащими ионообменник различной дисперсности. С увеличением времени измельчения ионообменных частиц, соответственно степени их дисперсности, выявлено уменьшение размеров проводящих участков и расстояния между ними на поверхности мембран. Высокая шумовая составляющая колебаний концентрационного поля в растворе вследствие развития электроконвективных вихрей установлена на границе с мембраной, характеризующейся более однородной поверхностью.

Ключевые слова: фликкер-шумовая спектроскопия, концентрационное поле, гетерогенная сульфокатионообменная мембрана, неоднородность поверхности, электроконвективная нестабильность, сверхпредельные токовые режимы
\end{abstract}

\section{Spectral properties of concentration field fluctuation in electromembrane systems with sulfocation-exchange membranes with different ion-exchanger dispersity}

\author{
Akberova E.M. \\ Voronezh State University, Voronezh
}

\begin{abstract}
One of the formation mechanisms of the concentration field oscillations in electromembrane systems under overlimiting current modes is electroconvective instability. The intensity of electroconvective mixing of the solution at the interface depends not only on current regimes and hydrodynamic conditions, but also is determined by heterogeneity of membrane surface. The aim of this work is to study the effect of surface properties of experimental samples of heterogeneous sulfocation-exchange membranes on the spectral composition of the concentration field fluctuations in solution under intensive current regimes.

The Fourier analysis method was used to determine the spectral composition of concentration field fluctuations in stratified systems with experimental heterogeneous sulfocation-exchange membranes Ralex CM Pes ( «MEGA»a.s., Czech Republic) containing an ion-exchanger of various dispersity. The degree of dispersity of the sulfocation-exchanger was varied by using a milling time from 5 to 80 minutes. The experiments were performed in a seven-compartment electrodialyzer at its stable concentration-temperature stratification in a gravitational field. To visualize the concentration fields at the membrane - solution boundary, a set up based on a Mach-Zehnder interferometer was used. The spectral density of the optical noise was calculated based on the Fast Fourier Transformation of time series of interference fringe fluctuations.
\end{abstract}


Using scanning electron microscopy, it was established a decrease in the spacing of electrical heterogeneity (the total size of the conductive and non-conductive regions) of the membrane surface with an increase in the milling time of ion-exchange particles from 5 to 80 minutes. Visualization by laser interferometry showed that reducing the spacing of the electrical surface heterogeneity leads to an increase in the intensity of the electroconvective solution instability at the interface. For experimental samples of CM Pes membranes, it was found increasing amplitude and frequency of oscillations of the interference band with increasing current density. The maximum values of the amplitude and average frequency of oscillations of the interference band, as well as the highest degree of electroconvective mixing of the solution, were observed at the interface with the membrane with a more homogeneous surface, i.e. containing the ionexchanger after 80 minutes of milling.

Keywords: flicker-noise spectroscopy, concentration field, heterogeneous sulfocation-exchange membrane, surface heterogeneity, electroconvective instability, overlimiting current modes

\section{Введение}

Значимым фактором, влияющим на интенсивность электроконвективной нестабильности в электромембранных системах при интенсивных токовых режимах, является электрическая (чередование проводящих и непроводящих участков) и геометрическая неоднородность (микрорельеф) поверхности мембран [1-7]. В случае мембран с неравномерной электрической проводимостью поверхности и шероховатостью происходит сгущение линий тока вблизи проводящих участков («эффект воронки» [8]) и появляется необходимая для развития интенсивной электроконвекции тангенциальная составляющая электрической силы [8-11]. Анализ флуктуационной динамики концентрационного поля (интерференционных полос) дает возможность получить информацию об особенностях конвективных потоков в растворе на границе с мембраной [12-15]. Целью настоящей работы явилось исследование влияния свойств поверхности экспериментальных образцов гетерогенных сульфокатионообменных мембран на спектральный состав флуктуаций концентрационного поля в растворе при интенсивных токовых режимах.

\section{Эксперимент}

Объектами исследования были выбраны экспериментальные гетерогенные ионообменные мембраны Ralex CM Pes («MEGA» a.s., Чехия), являющиеся композитом сульфокатионообменника с полиэтиленом и армирующей тканью полиэстер [16]. Экспериментальные образцы мембран были получены вальцеванием гомогенизированной смеси измельченного ионообменника (58\%) различной степени дисперсности с полиэтиленом. Степень дисперсности ионообменных частиц изменялась путем варьирования времени их измельчения от 5 до 80 мин. Образцы исследуемых мембран подвергали стандартной солевой предподготовке [17].

Эксперименты по изучению возникновения и развития концентрационных полей на межфазной границе при интенсивных токовых режимах были выполнены в семисекционной электродиализной ячейке. Исследуемый мембранный канал состоял из однотипных сульфокатионообменных мембран. Высота мембранного канала $L$ составляла $4.1 \cdot 10^{-2} \mathrm{M}$, ширина $1.4 \cdot 10^{-2} \mathrm{M}$, межмембранное расстояние $h=2.0 \cdot 10^{-3} \mathrm{M}$. Раствор $0.02 \mathrm{M}$ хлорида натрия в исследуемую секцию подавался со скоростью $1.3 \cdot 10^{-3}$ м $(\operatorname{Re}=2.6)$. Электродиализ был проведен в гальваностатическом режиме при горизонтальной ориентации электродиализатора в гравитационном поле (устойчивая концентрационно-температурная стратификация).

Для визуализации гидродинамических явлений в электромембранных системах использовали установку по схеме Маха-Цендера. Интерференционная картина регистрировалась на координате по направлению подачи раствора 
$y=2.6 \cdot 10^{-2} \mathrm{M}(0.64 \mathrm{~L})$ видеокамерой с частотой дискретизации 15 Гц и затем была представлена в цифровом виде. Результаты измерений флуктуаций интерференционных полос записывали в виде временных рядов длиной (2-3) $10^{3}$ отсчетов.

При исследовании оптических шумов применялся Фурье-анализ, позволивший сделать заключение о частотном составе шума, усредненного по всему времени записи колебательного процесса. Первичные данные, представляющие собой функцию времени, после обработки с помощью быстрого преобразования Фурье были получены в виде набора частот. Для оценки энергетического вклада различных частотных компонент был использован Фурье-спектр мощности, который для сигналов, имеющих шумовую природу с непрерывным спектром и содержащим широкий набор частотных компонент, состоит из участков вида $P \sim f^{-n}$, являющихся линейными функциями в двойных логарифмических координатах с коэффициентом наклона $n$.

Предварительно методом вольтамперных кривых были определены предельные диффузионные плотности тока в 0.02 М растворе хлорида натрия, которые составили $1.73,1.75$ и $1.81 \mathrm{MA} / \mathrm{cm}^{2}$ для экспериментальных мембран CM Pes co временем измельчения ионообменника 5, 40 и 80 мин, соответственно.

Исследования морфологии поверхности набухших мембран проводили методом растровой электронной микроскопии с применением микроскопа марки JSM6380 LV (Япония) с регулируемым давлением в камере с исследуемым образцом [18, 19]. Количественная оценка доли и размеров проводящих участков и макропор на поверхности мембран проводилась с помощью авторского программного комплекса [20]. При вычислении среднего радиуса микрофаз брали средневзвешенное значение [18], учитывающее различную долю участков с отличающимися размерами.

\section{Обсуждение результатов}

Микроскопическими методами выявлены различия свойств поверхности экспериментальных сульфокатионообменных мембран CM Pes. C ростом времени измельчения частиц ионообменника от 5 до 80 мин его доля на поверхности мембран сохраняется практически постоянной (табл. 1). При этом размеры проводящих участков уменьшались, микрорельеф поверхности становился более гладким. В выбранном диапазоне времени измельчения средняя величина шага неоднородности поверхности $2 \bar{R}+\bar{l}$, учитывающая изменение размеров ионообменников и расстояния между ними, линейно уменьшается от 13.0 мкм до 8.5 мкм. Наиболее однородным распределением характеристик поверхности обладает мембрана, полученная при времени измельчения ионообменника 80 мин.

Таблица 1. Характеристики поверхности гетерогенных сульфокатионообменных мембран Ralex CM Pes в набухшем состоянии: $S$ - доля частиц ионита; $\bar{R}$ - средневзвешенная величина радиуса частиц ионита; $P$ - пористость; $\bar{r}-$ средневзвешенная величина радиуса пор; $\bar{l}$ - размер непроводящего участка

\begin{tabular}{|c|c|c|c|c|c|}
\hline $\begin{array}{c}\text { Время измельчения, } \\
\text { мин }\end{array}$ & $S, \%$ & $\bar{R}$, мкм & $P, \%$ & $\bar{r}$, мкм & $\begin{array}{c}\bar{l}, \\
\text { мкм }\end{array}$ \\
\hline 5 & $16.6 \pm 0.7$ & $1.85 \pm 0.07$ & $2.7 \pm 0.2$ & $1.55 \pm 0.03$ & $9.3 \pm 0.8$ \\
\hline 40 & $16.8 \pm 0.5$ & $1.76 \pm 0.06$ & $2.4 \pm 0.3$ & $1.43 \pm 0.05$ & $6.4 \pm 0.6$ \\
\hline 80 & $15.4 \pm 0.6$ & $1.52 \pm 0.03$ & $1.8 \pm 0.1$ & $1.27 \pm 0.04$ & $5.5 \pm 0.4$ \\
\hline
\end{tabular}

Результаты измерения толщины области электроконвективной нестабильности (рис. 1) свидетельствуют о более интенсивном перемешивании раствора на гра- 
нице с экспериментальными мембранами, обладающими более однородной поверхностью. С уменьшением шага электрической неоднородности поверхности электроконвективная нестабильность возникает при меньших потенциалах, а область нестабильности имеет большие размеры. Вероятно, что в этом диапазоне изменения неоднородности поверхности мембран, сближение проводящей и непроводящей зон (частое чередование частиц ионообменника и полиэтилена) облегчает слияние соседних вихрей и усиливает электроконвективное перемешивание $[6,23,24]$, которое растет с увеличением скачка потенциала на мембране. Рост области конвективной нестабильности раствора у поверхности мембраны сопровождается уменьшением общей и эффективной толщин диффузионного слоя [25-27].

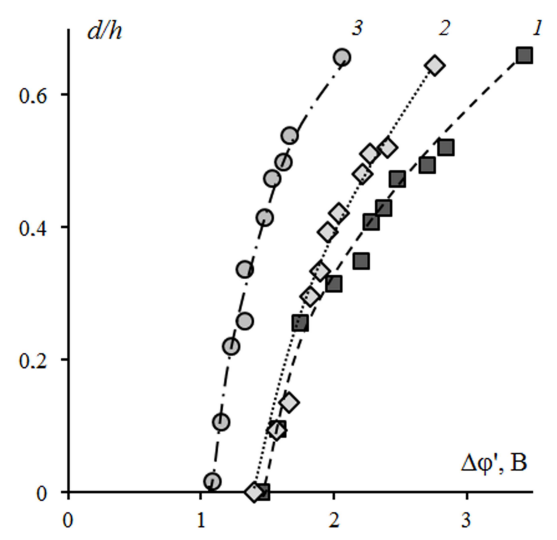

Рис. 1. Толщина области конвективной нестабильности $d$ в растворе на границе с катионообменными мембранами СM Pes со временем измельчения ионообменника 5 (1), 40 (2) и 80 (3) мин: $C_{0}(\mathrm{NaCl})=2.0 \cdot 10^{-2} \mathrm{M}, V=1.3 \cdot 10^{-3} \mathrm{M} / \mathrm{c}(\mathrm{Re}=2.6)$, межмембранное расстояние $h=2.0 \cdot 10^{-3} \mathrm{M}, y=2.6 \cdot 10^{-2} \mathrm{M}(0.64 \mathrm{~L})$. Величина приведенного скачка потенциала $\Delta \varphi^{\prime}$ рассчитана по выражению $\Delta \varphi^{\prime}=\Delta \varphi-i R_{\mathrm{ef}}[21,22]$.

Для экспериментальных образцов сульфокатионообменных мембран CM Pes с разным временем измельчения ионообменника установлено, что с ростом плотности тока увеличивались амплитуда и частота колебаний интерференционной полосы. Рост амплитуды флуктуаций потенциала с увеличением плотности тока на хронопотенциограммах ионообменных мембран в условиях, когда гравитационная конвекция подавлена, выявлен в работе [28]. Для экспериментальных мембран установлены заметные различия флуктуационной динамики концентрационного поля: в диапазоне токов $5.2<i / i_{l i m}<12.5$ амплитуда колебаний интерференционной полосы для мембраны со временем измельчения ионообменника 80 мин превышала соответствующую величину для образца, содержащего ионообменник после 5 мин измельчения, в 3.5 раза.

На рис. 2а представлены спектры мощности шума для экспериментальных образцов мембраны CM Pes при 5-кратном увеличении предельной диффузионной плотности тока. Установлено, что функция спектральной плотности имела $n$ характерную «фликкер-шумовую» зависимость вида $P \sim f^{-n}$. Изучение спектрального состава флуктуаций интерференционных полос выявило увеличение характеристического наклона участка спектра и возрастание показателя степени $n$ при увеличении кратности превышения предельной плотности тока для всех исследованных образцов сульфокатионообменных мембран. Данные по анализу флуктуаций мембранного потенциала с характерной для фликкер-шума частотной зависимостью $P \sim f^{-n}$, где $n$ 1-2 [29-31], 2.3-2.7 [32] и 3.0 [33], подтверждают возможность возрастания $n$ до измеренных значений при увеличении плотности тока в электромембранной системе. 
В рамках концепции фликкер-шумовой спектроскопии, возрастание показателя степени $n$ в спектре мощности шума интерференционной полосы отражало постепенный переход характера движения жидкости от ламинарного к предельно турбулизованному [34] по мере увеличения плотности тока. Максимальное значение $n=$ 3.0 для показателя степени в спектре мощности шума характеризует хаотическое объемное турбулентное перемешивание раствора. Сравнение величин параметра $n$ флуктуаций интерференционных полос у поверхности мембран CM Pes c разным временем измельчения приведено на рис. 2б. Доказательством более высокой интенсивности перемешивания раствора на границе с сульфокатионообменной мембраной с максимальным временем измельчения (80 мин) является возрастание параметра $n$ на $30 \%$ по сравнению с образцом мембраны, содержащим ионообменные частицы после 5 мин измельчения.
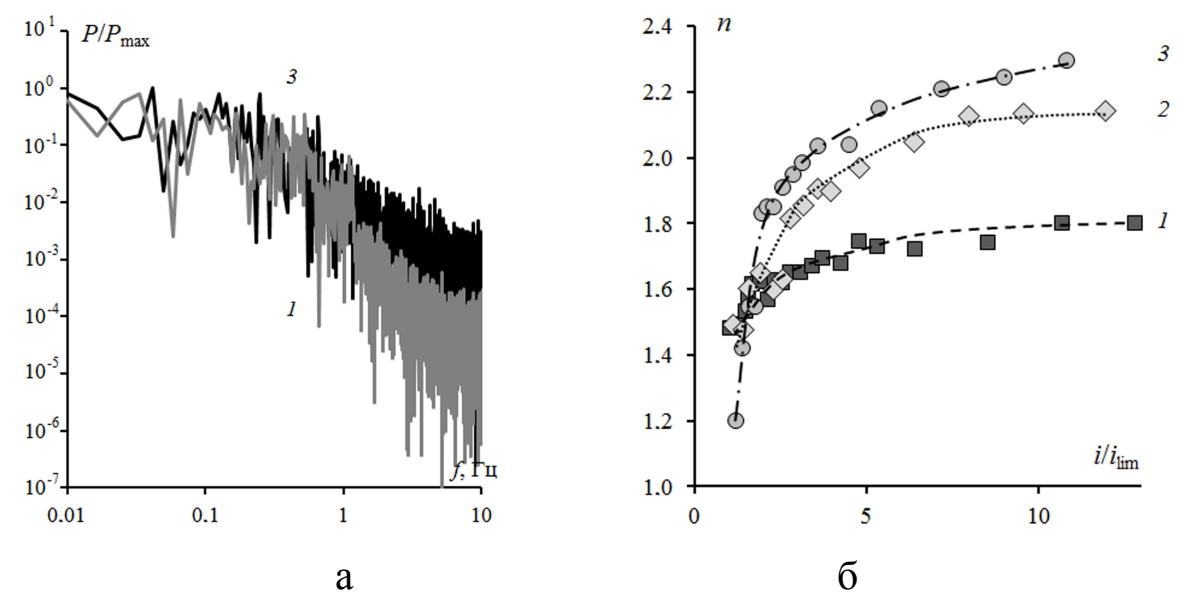

Рис. 2. Сравнение спектров мощности ( $a$ ) и параметра $n$ (б) флуктуаций интерференционных полос у поверхности катионообменных мембран CM Pes при $C_{0}(\mathrm{NaCl})=2.0 \cdot 10^{-2} \mathrm{M}, V=1.3 \cdot 10^{-3} \mathrm{M} / \mathrm{c}, h=2.0 \cdot 10^{-3}$ м. Время измельчения ионообменника, мин.: 5 (1), 40 (2) и 80 (3).

\section{Заключение}

Методом Фурье-анализа определен спектральный состав флуктуаций концентрационного поля в системах с экспериментальными сульфокатионообменными мембранами Ralex CM Pes. Методом растровой электронной микроскопии установлено уменьшение шага электрической неоднородности (суммарного размера проводящего и непроводящего участков) поверхности мембран с увеличением продолжительности измельчения ионообменных частиц с 5 до 80 мин. Визуализация методом лазерной интерферометрии показала, что уменьшение шага электрической неоднородности поверхности приводит к росту интенсивности электроконвективной нестабильности раствора. Максимальные значения амплитуды и средней частоты колебаний интерференционной полосы, а также наибольшая степень электроконвективного перемешивания раствора наблюдались на границе с мембраной с более однородной поверхностью, т.е. содержащей ионообменник после 80 мин измельчения.

Работа выполнена при поддержке гранта Президента Российской Федераџии MK-925.2018.3.

Автор выражсает благодарность компании «Меgа» а.s. (Чешская Республика) и г-ну Новаку Л. за предоставленные экспериментальные мембраны Ralex CM Pes.

Микрофотографии поверхности мембран получены на оборудовании Центра 
коллективного пользования Воронежского государственного университета. URL: http://ckp.vsu.ru.

\section{Список литературы}

1. Заболоцкий В.И., Новак Л., Коваленко А.В., Никоненко В.В. и др. // Мембраны и мембранные технологии. 2017. Т. 7. № 4. С. 265-276.

2. Заболоцкий В.И., Никоненко В.В., Уртенов М.Х., Лебедев К.А. и др. // Электрохимия. 2012. Т.48. №7. С. 766-777.

3. Никоненко В.В., Мареев С.А., Письменская Н.Д., Узденова А.М. и др. // Электрохимия. 2017. Т. 53. № 10. С. 1266-1289.

4. Nikonenko V. V., Pismenskaya N. D., Belova E. I., Sistat Ph. et al.// Adv. Colloid Interface Sci. 2010. Vol. 160. Iss. 1-2. pp. 101123.

5. Васильева В.И., Акберова Э.М., Заболоцкий В.И. // Электрохимия. 2017. Т. 53. № 4. С. 452-465.

6. Васильева В. И., Жильцова А.В., Акберова Э.М., Фатаева А.И. // Конденсированные среды и межфазные границы. 2014. Т. 16. № 3. C. 257-261.

7. Княгиничева Е.В., Белашова Е.Д., Сарапулова В.В., Письменская Н.Д. // Конденсированные среды и межфазные гранищы. 2014. T. 16. № 3. C. 282-287.

8. Rubinstein I., Zaltzman B., Kedem O. // J. Memb. Sci. 1997. Vol. 125. pp. 17-21.

9. Dukhin S.S., Mishchuk N.A. // J. Membr. Sci. 1993. Vol. 79. pp. 199-210.

10. Rubinstein I., Zaltzman B., Pundik T. // Phys. Rev. E. 2002. Vol. 65. pp. 41507.

11. Mishchuk N.A. // Curr. Opin. Colloid Interface Sci. 2013. Vol. 18. pp. 137-148.

12. Васильева В.И., Жильцова А.В., Малыхин М.Д., Григорчук О.В. и др. // Сорбиионные и хроматографические прочессы. 2009. T.9. № 2. С. 196-207.

13. Васильева В.И., Григорчук О.В., Ботова Т.С., Заболоцкий В.И. и др. // Сорбционные и хроматографические прочессы. 2008. Т.8. № 3. С. 359-379.

14. Жильцова А.В., Малыхин М.Д., Васильева В.И. // Сорбиионные и хроматографические проиессы. 2009. Т.9. № 6. С. 904915.

15. Vasi1'eva V.I., Shaposhnik V.A., Zhiltsova A.V., Grigorchuk O. et al. // Desalination and water treatment. 2010. Vo1. 14. pp. 214219.
16. Сайт

«MEGA

a.s.»

https://www.mega.cz/ (дата обращения

19.10.2018)

17. Березина Н.П., Кононенко Н.А., Дворкина Г.А, Шельдешов Н.В. Физикохимические свойства ионообменных материалов. Краснодар. Изд-во Кубан. гос. ун-та. 1999. $82 \mathrm{c}$.

18. Васильева В.И., Акберова Э.М., Жильцова А.В., Черных Е.И. и др. // Поверхность. Рентгеновские, синхротронные и нейтронные исследования, 2013. № 9. С. 27-34.

19. Васильева В.И., Письменская Н.Д., Акберова Э.М., Небавская К.А. // Журн. физ. химии. 2014. Т. 88. № 8. С. 1114-1120.

20. Сирота Е.А., Кранина Н.А., Васильева В.И., Малыхин М.Д. и др. // Вестник Воронежского государственного университета. Серия: Химия. Биология. Фармачия. 2011. № 2. C. 53-59.

21. Maletzki F., Rosler H.-W., Staude E.J. // J. Membr. Sci. 1992. Vol. 71. pp. 105-116.

22. Письменская Н.Д., Никоненко В.В., Белова Е.И., Лопаткова Г.Ю. и др. // Электрохимия. 2007. Т 43. С. 325-345.

23. Akberova E.M., Vasil'eva V.I., Zabolotsky V.I., Novak L. // Journal of Membrane Science. 2018. Vol. 566. pp. 317-328.

24. Васильева В.И., Акберова Э.М., Заболоцкий В.И., Новак Л. И др. // Мембраны и мембранные технологии. 2018. Т. 8. № 6. С. 411-422.

25. Nikonenko V.V., Vasil'eva V.I., Akberova E.M., Uzdenova A.M. et al. // $A d v$. Colloid Interface Sci. 2016. Vol. 235. pp. 233246.

26. Акберова Э.М. // Конденсированные среды и межфазные гранииы. 2017. Т. 19. № 3. C. 314-320.

27. Акберова Э.М., Костылев Д.В., Васильева В.И. // Конденсированные среды и межфазные гранииьы. 2018. Т. 20. № 3. С. 354-363.

28. Krol J.J., Wessling M., Strathmann H. // J. Membr. Sci. 1999. Vol. 162. pp. 155-164.

29. Fang Y., Li Q., Green M.E. // J. Colloid. Interface Sci. 1982. Vol. 86. No 1. pp. 185-190. 
30. Колюбин А.В., Максимычев А.В., Тимашев С.Ф. // Электрохимия. 1996. Т.32. № 2. С. 227-234.

31. Green M.F., Yafusso M. // J. Phys. Chem. 1968. Vol.72. pp. 4072-4078.

32. Тимашев С.Ф., Григорьев В.В., Будников Е.Ю. // Журн. физ. химии. 2002. Т. 76. № 3. С. 554-561.

\section{References}

1. Zabolotsky V.I., Novak L., Kovalenko A.V., Nikonenko V.V. et al., Petroleum Chemistry, 2017, Vol. 57, Iss. 9, pp. 779-789.

2. Zabolotskii V.I., Nikonenko V.V., Urtenov M.Kh., Lebedev K.A. et al., Russ. J. Electrochem., 2012, Vol. 48, No 7, pp. 692-703.

3. Nikonenko V.V., Mareev S.A., Pis'menskaya N.D., Uzdenova A.M. et al., Russ. J. Electrochem., 2017, Vol. 53, Iss. 10, pp. 1122-1144.

4. Nikonenko V.V., Pismenskaya N.D., Belova E.I., Sistat Ph. et al., Adv. Colloid Interface Sci., 2010, Vol. 160, Iss. 1-2, pp. 101-123.

5. Vasil'eva V. I., Akberova E. M., Zabolotskii V. I., Russ. J. Electrochem., 2017, Vol. 53, No 4. pp. 398-410.

6. Vasil'eva V.I., Zhil'tsova A.V., Akberova E.M., Fataeva A.I., Kondensirovannye sredy $i$ mezhfaznye granitsy, 2014, Vol. 16, No 3, pp. 257-261.

7. Knyaginicheva E.V., Belashova E.D., Sarapulova V.V., Pismenskaya N.D., Kondensirovannyye sredy $i$ mezhfaznyye granitsy, 2014, Vol. 16, No 3, pp. 282-287.

8. Rubinstein I., Zaltzman B., Kedem O., J. Memb. Sci., 1997, Vol. 125, pp.17-21.

9. Dukhin S.S., Mishchuk N.A., J. Membr. Sci., 1993, Vol. 79, pp. 199-210.

10. Rubinstein I., Zaltzman B., Pundik T., Phys. Rev. E., 2002, Vol. 65, pp. 41507.

11. Mishchuk N.A., Curr. Opin. Colloid Interface Sci., 2013, Vol. 18, pp. 137-148.

12. Vasil'eva V.I., Zhiltsova A.V., Malykhin M.D., Grigorchuk O.V. et al., Sorbtsionnyye $i$ khromatograficheskiye protsessy, 2009, Vol. 9, No 2, pp. 196-207.

13. Vasil'eva V.I., Grigorchuk O.V., Botova T.S., Zabolotskii V.I. et al., Sorbtsionnye $i$ khromatograficheskie protsessy, 2008, Vol. 8, No 3, pp. 359-379.

14. Zhiltsova A.V., Malykhin M.D., Vasil'eva V.I., Sorbtsionnye $i$ khromatograficheskie protsessy, 2009, Vol. 9, No 6, pp. 904915.
33. Будников Е.Ю., Максимычев А.В., Колюбин А.В., Тимашев С.Ф. // Электрохимия. 2001. T.37. № 1. С. 95-103.

34. Тимашев С. Ф. Фликкер-шумовая спектроскопия: информация в хаотических сигналах. М. ФИЗМАТЛИТ. 2007. 248 с.

15. Vasil'eva V.I., Shaposhnik V.A., Zhiltsova A.V., Grigorchuk O. et al., Desalination and water treatment, 2010, Vo1. 14, pp. 214219.

16. «MEGA a.s.» web-site https://www.mega.cz/ (accessed19.10.2018)

17. Berezina N.P., Kononenko N.A., Dvorkina G.A, Sheldeshov N.V. Fiziko-khimicheskiye svoystva ionoobmennykh materialov. Krasnodar: Kuban State Univ. Pub., 1999, 82 p.

18. Vasil'eva V.I., Akberova E.M., Zhiltsova A.V., Chernykh E. I. et al., J. Surf. Investigation. Xray, Synchrotron and Neutron Techniques, 2013, Vol. 7, No 5, pp. 833-840.

19. Vasil'eva V.I., Pismenskaya N.D., Akberova E.M., Nebavskaya K.A., Russ. J. Phys. Chem. A, 2014, Vol. 88, No. 8, pp. 1293-1299.

20. Sirota E.A., Kranina N.A., Vasil'eva V.I., Malykhin M.D., Selemenev V.F., Vestnik Voronezhskogo gosudarstvennogo universiteta. Seriya: Khimiya. Biologiya. Farmatsiya, 2011, No 2, pp. 53-59.

21. Maletzki F., Rosler H.-W., Staude E.J., J. Membr. Sci., 1992, Vol. 71, pp. 105-116.

22. Pismenskaya N.D., Nikonenko V.V., Belova E.I., Lopatkova G.Yu., Sistat Ph., Pourcelly G., Larshe K., Russ. J. Electrochem., 2007, Vol. 43, pp. 307-327.

23. Akberova E.M., Vasil'eva V.I., Zabolotsky V.I., Novak L., J. Membr. Sci., 2018, Vol. 566, pp. 317-328.

24. Vasil'eva V.I., Akberova E.M., Zabolotsky V.I., Novak L. et al., Petroleum Chemistry, 2018, Vol. 58, No 13, pp. 1133-1143.

25. Nikonenko V.V., Vasil'eva V.I., Akberova E.M., Uzdenova A.M. et al., Adv. Colloid Interface Sci., 2016, Vol. 235, pp. 233-246.

26. Akberova E. M., Kondensirovannye sredy i mezhfaznye granitsy, 2017, Vol. 19, No 3, pp. 314-320.

27. Akberova E. M., Kostylev D.V., Vasil'eva V.I., Kondensirovannye sredy $i$ mezhfaznye granitsy, 2018, Vol. 20. No 3, pp. 354-363. 
28. Krol J.J., Wessling M., Strathmann H., J. Membr. Sci., 1999, Vol.162, pp. 155-164.

29. Fang Y., Li Q., Green M.E., J. Colloid. Interface Sci., 1982, Vol. 86, No 1, pp. 185-190.

30. Kolyubin A.V., Maksimychev A.V., Timashev S.F., Elektrokhimiya, 1996, Vol. 32, No 2, pp. 227-234.

31. Green M.F., Yafusso M., J. Phys. Chem., 1968, Vol.72, pp. 4072-4078.

Акберова Эльмара Маликовна - к.х.н., ведущий инженер кафедры аналитической химии, Воронежский государственный университет, Воронеж.
32. Timashev S.F., Grigor'yev V.V., Budnikov E.Yu., Zhurnal fizicheskoy khimii, 2002, Vol. 76, No 3, pp. 554-561.

33. Budnikov E.Yu., Maksimychev A.V., Kolyubin A.V., Timashev S.F., Russ. J. Electrochem., 2001, Vol. 37, No 1. pp. 80-87.

34. Timashev S.F. Flikker-shumovaya spektroskopiya: informatsiya $\mathrm{v}$ khaoticheskikh signalakh. Moscow: FIZMATLIT, 2007, 248p.

Akberova Elmara M. - PhD (Chem.), leading engineer of the Analytical Chemistry Department, Voronezh State University, Voronezh, e-mail: elmara_09@inbox.ru. 